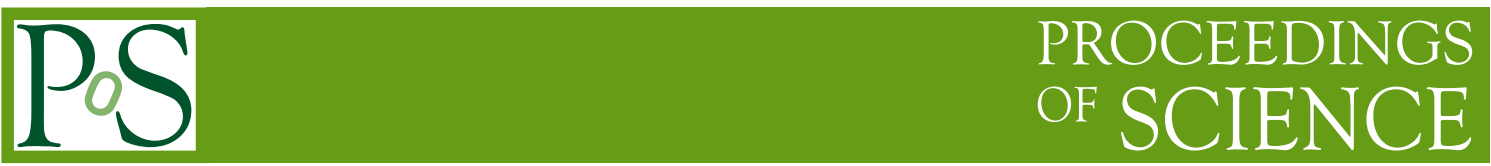

\title{
Neutrino Portals to Dark Matter
}

\section{Salvador Rosauro-Alcaraz ${ }^{a, *}$ \\ ${ }^{a}$ Departamento de Física Teórica and Instituto de Física Teórica, IFT-UAM/CSIC, Universidad Autónoma de Madrid, Cantoblanco, 28049, Madrid, Spain}

E-mail: salvador.rosauro@uam.es

We explore the possibility that dark matter interactions with Standard Model particles are dominated by interactions with neutrinos. We examine whether it is possible to construct such a scenario in a gauge invariant manner. We first study the coupling of dark matter to the full lepton doublet and confirm that this generally leads to the dark matter phenomenology being dominated by interactions with charged leptons. We then explore two different implementations of the neutrino portal in which neutrinos mix with a Standard Model singlet fermion that interacts directly with dark matter through either a scalar or vector mediator. In the latter cases we find that the neutrino interactions can dominate the dark matter phenomenology. Present neutrino detectors can probe dark matter annihilations into neutrinos and already set the strongest constraints on these realisations. Future experiments such as Hyper-Kamiokande, MEMPHYS, DUNE, or DARWIN could allow to probe dark matter-neutrino cross sections down to the value required to obtain the correct thermal relic abundance.

40th International Conference on High Energy physics - ICHEP2020

July 28 - August 6, 2020

Prague, Czech Republic (virtual meeting)

\footnotetext{
${ }^{*}$ Speaker
} 

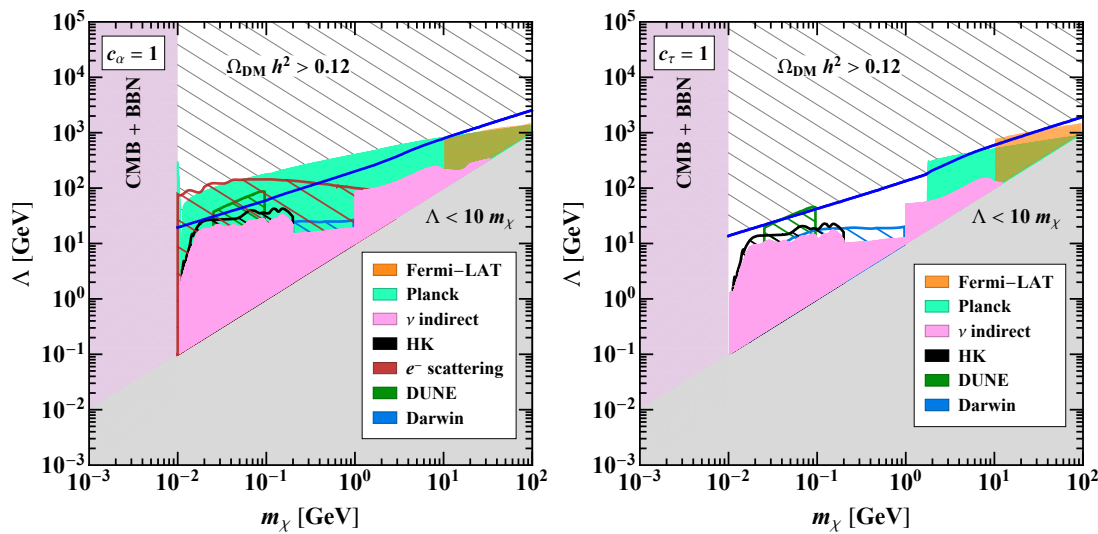

Figure 1: Constraints on the DM mass $m_{\chi}$ and the new physics scale $\Lambda$. The left (right) panel corresponds to coupling to every lepton (only tau) doublets.

\section{Introduction}

The unknown origin of neutrino masses together with the existence of the dark matter (DM) component of the Universe constitute our most significant experimental evidence for physics beyond the Standard Model (SM) and therefore the best windows to explore new physics. Neutrinos and DM also share their elusive nature, having very weak interactions with the other SM particles. A tantalising avenue of investigation is the possibility that a stronger connection between these two sectors exists. In this case, the best way to probe DM would be through the neutrino sector.

In this contribution, based on [1], we will investigate some gauge-invariant SM extensions that lead to sizeable neutrino-DM interactions, exploring if neutrino probes could dominate our sensitivity to the dark sector. If DM does not participate in any of the SM gauge interactions, the natural expectation is that the strongest connection to DM will be via singlets of the SM gauge group. The neutrino portal includes the addition of singlet right-handed (RH) neutrinos $N_{R}$, which makes this option particularly appealing in connection to the evidence of neutrino masses and mixing.

\section{Coupling to the full lepton doublet}

In this section we will study the simplest scenario, in which DM couples directly to the full SM $S U(2)$ lepton doublet. We will adopt an effective field theory approach in order to avoid specifying the nature of the mediator. The Lagrangian describing the neutrino-DM interaction is thus given by

$$
\mathcal{L}=\mathcal{L}_{S M}+\mathcal{L}_{D M}+\frac{c_{\alpha}}{\Lambda^{2}} \bar{\chi} \gamma_{\mu} \chi \overline{L_{\alpha}} \gamma^{\mu} L_{\alpha}
$$

with $\mathcal{L}_{D M}=\bar{\chi}\left(i \not \supset-m_{\chi}\right) \chi$, being $\chi$ a Dirac fermion DM particle. From Eq. (1) it is clear that DM couples as strongly to neutrinos as to charged leptons.

As can be seen in Fig. 1, whenever the annihilation to the corresponding charged lepton is possible, the constraints from Fermi-LAT [2] and the CMB [3, 4] dominate, ruling out all the parameter space. Only if $m_{\chi}$ is smaller than the mass of the corresponding charged lepton do neutrino experiments [5-10] control the sensitivity to these interactions. Future neutrino and DM 

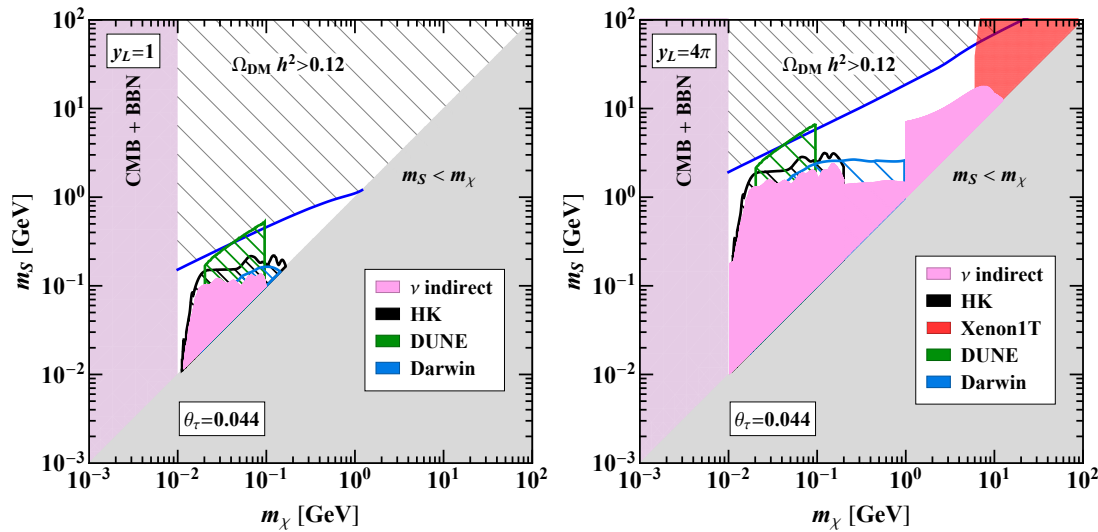

Figure 2: Constraints on the DM mass $m_{\chi}$ and the dark scalar mass $m_{S}$. We have fixed $\theta_{\tau}=0.044, \theta_{e, \mu}=0$. Along the blue line the DM relic density matches the observed value. The coloured shaded regions are excluded by different experiments, while the hatched areas correspond to prospective sensitivities of future experiments.

experiments [11-15] could further explore these unconstrained regions as shown in the right panel of Fig. 1. For more details see Ref. [1].

\section{Coupling via the neutrino portal}

Given the results of the previous section, we will now explore whether the neutrino portal option is able to lead to a rich DM-neutrino phenomenology without being in conflict with indirect searches involving charged leptons. In order to have sizeable mixing between the SM neutrinos and the extra $N_{R}$ we will attribute the smallness of neutrino masses to an approximate lepton number symmetry [16-18]. We will consider the addition of only one Dirac sterile neutrino that will serve as portal between the SM neutrinos and the DM. The Lagrangian of the model is given by

$$
\mathcal{L}_{N}=\mathcal{L}_{S M}+\bar{N}\left(i \not \partial-m_{N}\right) N-\lambda_{\alpha} \overline{L_{\alpha}} \tilde{H} N_{R}
$$

After electroweak symmetry breaking we get three massless neutrinos and a heavy one with mass $m_{4}=\sqrt{m_{N}^{2}+\sum_{\alpha} v^{2}\left|\lambda_{\alpha}\right|^{2}}$. The amount of mixing between SM and the singlet neutrinos will be controlled by the size of $\theta_{\alpha} \equiv v \lambda_{\alpha} / m_{N}$

\subsection{Neutrino portal with a scalar mediator}

In this section we will assume that DM is composed of a new fermion, singlet under the SM gauge group, and that a new scalar mediates the neutrino-DM interactions. The Lagrangian of the model is given by

$$
\mathcal{L}=\mathcal{L}_{N}+\mathcal{L}_{D M}+\partial_{\mu} S \partial^{\mu} S^{*}-y_{L} \bar{\chi} N_{L} S+\text { h.c. }-V\left(|S|^{2}, H^{\dagger} H\right),
$$

where $S$ is a complex scalar and $\chi$ is a Dirac fermion DM candidate. Note that the Lagrangian respects $U(1)_{L}$ lepton number and a global dark symmetry under which $\chi$ and $S$ have the same charge. In order to have DM stability we will assume $m_{\chi}<m_{S}$, where $m_{S}$ is the mass of the scalar. 

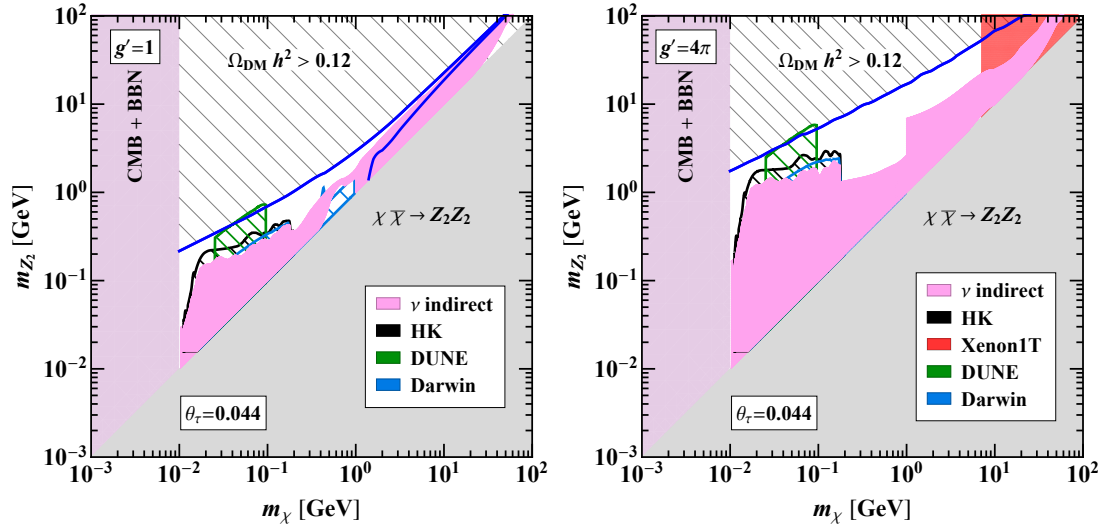

Figure 3: Constraints on the DM mass $m_{\chi}$ and $m_{Z_{2}}$ for $\theta_{\tau}=0.044, \theta_{e, \mu}=0$. Along the blue line the DM relic density matches the observed value. The coloured shaded regions are excluded by different experiments, while the hatched areas correspond to prospective sensitivities of future experiments.

The sensitivity to these interactions will be controlled by neutrino experiments, as the coupling to other SM particles appears at loop level.

Along the blue line in Fig. 2 the measured DM relic density [3] is obtained. Above this line the relic density is larger, leading to overclosure of the Universe. Indirect searches for annihilation to neutrinos, together with direct detection bounds by XENON1T [19], are the only probes that constrain the allowed parameter space. The prospects to explore the remaining allowed regions are very promising (see Ref. [1] ).

\subsection{Neutrino portal with a vector mediator}

In this second example, we will couple the Dirac DM fermion to a new massive vector boson. The Lagrangian of the model is given by

$$
\mathcal{L}=\mathcal{L}_{N}+\mathcal{L}_{D M}-\frac{1}{4} Z_{\mu \nu}^{\prime} Z^{\prime \mu \nu}+\frac{1}{2} m_{Z^{\prime}}^{2} Z_{\mu}^{\prime} Z^{\prime \mu}+g^{\prime} \overline{\chi_{R}} \gamma^{\mu} \chi_{R} Z_{\mu}^{\prime}+g^{\prime} \overline{N_{L}} \gamma^{\mu} N_{L} Z_{\mu}^{\prime}
$$

where $Z^{\prime}$ is a new vector boson mediating the interaction between neutrinos and DM. This Lagrangian could describe a new spontaneously broken $U(1)^{\prime}$ gauge symmetry. We will assume there is an additional conserved charge not shared between the neutrino and the DM preventing their mixing. The phenomenology will be dominated by neutrino experiments, as the coupling to other SM particles appears at loop level, e.g., through kinetic mixing between the $Z$ and $Z^{\prime}$ vector bosons [1].

In Fig. 3 there are two branches where the DM relic density is obtained due to a resonant behaviour of the annihilation cross section to neutrinos. Large regions of parameter space are constrained through indirect detection at neutrino detectors. Future neutrino experiments will further probe other regions of parameter space, with DUNE being able to probe the relic density target . 


\section{Conclusions}

We have explored whether a dominant neutrino-DM interaction is allowed in simple gaugeinvariant models. We first explored the simplest scenario, in which DM couples to the full lepton doublet. Whenever DM is heavier than the charged lepton(s) it couples to, the bounds from Fermi-LAT and CMB preclude DM-neutrino couplings sizeable enough to be probed.

We have then considered the option of the neutrino portal to DM, where DM couples directly to new heavy neutrinos, which are a natural addition to the SM to account for neutrino masses and mixing. In the two realisations we explored we find that neutrino detectors place the most stringent and competitive bounds. Future projects will be able to probe if the right-handed singlet fermions that can explain the origin of neutrino masses also represent our best window to the dark matter sector.

\section{References}

[1] M. Blennow, E. Fernandez-Martinez, A. Olivares-Del Campo, S. Pascoli, S. RosauroAlcaraz and A. Titov, Neutrino Portals to Dark Matter, Eur. Phys. J. C 79 (2019) 555 [arXiv: 1903.00006].

[2] MAGIC AND Fermi-LAT Collaboration, M. L. Ahnen et al., Limits to dark matter annihilation cross-section from a combined analysis of MAGIC and Fermi-LAT observations of dwarf satellite galaxies, JCAP 1602 (2016) 039 [arXiv: 1601.06590].

[3] Planck Collaboration, N. Aghanim et al., Planck 2018 results. VI. Cosmological parameters, arXiv: 1807.06209.

[4] T. R. Slatyer, Indirect dark matter signatures in the cosmic dark ages. I. Generalizing the bound on s-wave dark matter annihilation from Planck results, Phys. Rev. D93 (2016) 023527 [arXiv: 1506.03811].

[5] Borexino Collaboration, G. Bellini et al., Study of solar and other unknown anti-neutrino fluxes with Borexino at LNGS, Phys. Lett. B696 (2011) 191-196 [arXiv: 1010 . 0029].

[6] A. Olivares-Del Campo, C. Boehm, S. Palomares-Ruiz and S. Pascoli, Dark matter-neutrino interactions through the lens of their cosmological implications, Phys. Rev. D97 (2018) 075039 [arXiv: 1711.05283].

[7] J. F. Beacom, N. F. Bell and G. D. Mack, General Upper Bound on the Dark Matter Total Annihilation Cross Section, Phys. Rev. Lett. 99 (2007) 231301 [arXiv: astro-ph/0608090].

[8] FREJUS Collaboration, C. Berger et al., The Frejus Nucleon Decay Detector, Nucl. Instrum. Meth. A262 (1987) 463.

[9] AMANDA Collaboration, F. Halzen et al., The AMANDA neutrino telescope, Nucl. Phys. Proc. Suppl. 77 (1999) 474-485 [arXiv: hep-ex/9809025]. 
[10] Super-Kamiokande Collaboration, K. Frankiewicz, Searching for Dark Matter Annihilation into Neutrinos with Super-Kamiokande, in Proceedings, Meeting of the APS Division of Particles and Fields (DPF 2015): Ann Arbor, Michigan, USA, 4-8 Aug 2015, 2015 [arXiv: 1510.07999].

[11] Hyper-Kamiokande Collaboration, K. Abe et al., Hyper-Kamiokande Design Report, arXiv: 1805.04163.

[12] ESSNuSB Collaboration, E. Baussan et al., A very intense neutrino super beam experiment for leptonic CP violation discovery based on the European spallation source linac, Nucl. Phys. B885 (2014) 127-149 [arXiv: 1309 . 7022].

[13] A. Olivares-Del Campo, S. Palomares-Ruiz and S. Pascoli, Implications of a Dark MatterNeutrino Coupling at Hyper-Kamiokande, in 53rd Rencontres de Moriond on Electroweak Interactions and Unified Theories (Moriond EW 2018) La Thuile, Italy, March 10-17, 2018, 2018 [arXiv: 1805.09830].

[14] DARWIN Collaboration, J. Aalbers et al., DARWIN: towards the ultimate dark matter detector, JCAP 1611 (2016) 017 [arXiv: 1606.07001].

[15] DUNE Collaboration, R. Acciarri et al., Long-Baseline Neutrino Facility (LBNF) and Deep Underground Neutrino Experiment (DUNE), arXiv: 1512.06148.

[16] M. Malinsky, J. Romao and J. Valle, Novel supersymmetric SO(10) seesaw mechanism, Phys. Rev. Lett. 95 (2005) 161801 [arXiv: hep-ph/0506296].

[17] R. Mohapatra and J. Valle, Neutrino Mass and Baryon Number Nonconservation in Superstring Models, Phys. Rev. D34 (1986) 1642.

[18] J. Bernabeu, A. Santamaria, J. Vidal, A. Mendez and J. Valle, Lepton Flavor Nonconservation at High-Energies in a Superstring Inspired Standard Model, Phys. Lett. B187 (1987) 303.

[19] XENON Collaboration, E. Aprile et al., Dark Matter Search Results from a One Ton-Year Exposure of XENONIT, Phys. Rev. Lett. 121 (2018) 111302 [arXiv: 1805. 12562]. 